\title{
REMARKS ON BIMEASURES
}

\author{
IGOR KLUVÁNEK
}

ABSTRACT. Techniques of vector valued measures are used to obtain disintegrations of bimeasures.

Bimeasures are considered of little importance [1, p. 129] because any reasonably interesting nonnegative real valued bimeasure determines a measure in the product space [4]. However, when one considers bimeasures of arbitrary sign or, more generally, vector valued bimeasures this attitude is not justified: in a sense, only trivial cases of such bimeasures can be treated as measures. Moreover, in the writer's opinion, bimeasures represent an important, although as yet unexplored, tool for operator theory and related fields. Fruitful developments seem to have been impeded by the lack of techniques related to bimeasures. Indeed, a series of counterexamples in spectral theory, following that of S. Kakutani [2], indicate that this may be the case. At any rate, a majority of bimeasures occuring naturally in worthwhile problems are derived from some vector or operator valued measures, in particular spectral measures.

The purpose of this note is to contribute to the clarification of some basic phenomena related to bimeasures, in particular their structure. It should not be too surprising that many of these phenomena can be explained only if vector valued measures are drawn into the game. This also applies to the case of scalar valued bimeasures as demonstrated by Kari Ylinen [6] (see especially Theorem 4.4).

Let $\mathcal{S}$ be a $\sigma$-algebra of sets in a space $\Omega$. An $\mathcal{S}$-partition of a set $E \in \mathcal{S}$ is a finite collection of pair-wise disjoint sets from $\delta$ whose union is equal to $E$. The set of all $\delta$-partitions of the set $E$ is denoted by $\Pi(E ; \delta)$.

Let $X$ be a locally convex space. A map $m: \mathcal{S} \rightarrow X$ is called a measure if it is $\sigma$-additive.

The set of all measures $m: \mathcal{S} \rightarrow X$ is denoted by $\mathrm{ca}(\mathcal{S}, X)$. The set of all nonnegative measures belonging to $\mathrm{ca}(\mathcal{S}, \mathbf{R})$ is denoted by $\mathrm{ca}^{+}(\mathcal{S}, \mathbf{R})$, and the set of all nonnegative extended real valued measures on $\mathcal{S}$ by ca ${ }^{+}(\mathcal{S}, \overline{\mathbf{R}})$.

If $X$ is a Banach space and $m: \delta \rightarrow X$ a vector measure, the variation of $m$ is the smallest measure $\lambda \in \mathrm{ca}^{+}(\mathcal{S}, \overline{\mathbf{R}})$ such that $\|m(E)\|<\lambda(E)$, for every $E \in \mathcal{\delta}$.

A scalar valued function $f$ on $\Omega$ is said to be integrable with respect to a measure $m: \mathcal{S} \rightarrow X$ if there exists a unique measure $n: \mathcal{S} \rightarrow X$ such that

$$
n(E) \in \overline{\operatorname{co}}\left\{\sum_{G \in \mathscr{P}} f\left(\omega_{G}\right) m(G): \omega_{G} \in G, G \in \mathscr{P}, \mathscr{P} \in \Pi(E ; \delta)\right\},
$$

Received by the editors February 15, 1980.

AMS (MOS) subject classifications (1970). Primary 28A40, 28A50; Secondary 28A45.

Key words and phrases. Vector bimeasure, vector measure, disintegration of a bimeasure. 
for every $E \in \mathcal{S}$. The measure $n$ is then called the indefinite integral of $f$ with respect to $m$ and is denoted by $f m$. Of course, the standard notations

$$
(f m)(E)=\int_{E} f d m=\int_{E} f(\omega) m(d \omega)
$$

are also used.

Let $\delta$ and $\mathcal{T}$ be $\sigma$-algebras of sets in spaces $\Omega$ and $\Upsilon$, respectively. The family of sets $\{E \times F: E \in \mathcal{S}, F \in \mathcal{T}\}$ is denoted by $\mathcal{S} \times \mathcal{T}$. An $\mathcal{S} \times \mathcal{T}$-partition of a set $E \times F \in \mathcal{S} \times \mathcal{T}$ is a finite collection of pairwise disjoint sets from $\mathcal{S} \times \mathcal{T}$ whose union is $E \times F$. The set of all $\delta \times \mathcal{T}$-partitions of the set $E \times F$ is denoted by $\Pi(E \times F ; \mathcal{S} \times \mathcal{T})$. The $\sigma$-algebra generated by $\mathcal{S} \times \mathcal{T}$ is denoted by $\mathcal{S} \otimes \mathcal{T}$.

A map $b: \mathcal{\delta} \times \mathcal{T} \rightarrow X$ is called a bimeasure if, for every $E \in \mathcal{S}$, the map $F \mapsto b(E \times F), F \in \mathcal{T}$, is a measure and, for every $F \in \mathcal{T}$, the map $E \mapsto$ $b(E \times F), E \in \mathcal{S}$, is a measure.

By the restriction of a bimeasure $b: \mathcal{S} \times \mathcal{T} \rightarrow X$ to the set $E \times F, E \in \mathcal{S}$, $F \in \mathcal{T}$, we mean the bimeasure $E^{\prime} \times F^{\prime} \mapsto b\left(E^{\prime} \times F^{\prime}\right), E^{\prime} \in \mathcal{S}, E^{\prime} \subset E, F^{\prime} \in \mathcal{T}$, $F^{\prime} \subset F$.

A scalar function $f$ on $\Omega \times T$ is said to be integrable with respect to a bimeasure $b: \mathcal{S} \times \mathcal{T} \rightarrow X$ if there exits a unique bimeasure $c: \mathcal{S} \times \mathcal{T} \rightarrow X$ such that

$$
c(E \times F) \in \overline{c o}\left\{\sum_{G \in \mathcal{P}} f\left(\xi_{G}\right) b(G): \xi_{G} \in G, G \in \mathcal{P}, \mathcal{P} \in \Pi(E \times F ; \mathcal{S} \times \mathcal{T})\right\},
$$

for every $E \in \mathcal{S}$ and $F \in \mathcal{T}$. The bimeasure $c$ is then called the indefinite integral of the function $f$ with respect to the bimeasure $b$ and is denoted by $f b$.

In earlier literature, the integral with respect to a bimeasure is defined only for functions of the form $(\omega, v) \mapsto f(\omega) g(v),(\omega, v) \in \Omega \times \Upsilon$, or, alternatively, for couples $(f, g)$, where $f$ is a function on $\Omega$ and $g$ a function on $\Upsilon$. The literature on this subject is not extensive and the bibliography in Ylinen's paper [6] seems to cover it completely.

Many bimeasures can be obtained in the following way.

We take a locally convex space $X$, a measure $\lambda \in \mathrm{ca}^{+}(\mathcal{\delta}, \overline{\mathbf{R}})$ and, for every $\omega \in \Omega$, a measure $m(\omega) \in \operatorname{ca}(\mathcal{T}, X)$. The value of the measure $m(\omega)$ at a set $F \in \mathcal{T}$ is denoted by $m(\omega ; F)$. Then we put

$$
b(E, F)=\int_{E} m(\omega ; F) \lambda(d \omega),
$$

for every $E \in \mathcal{S}$ and $F \in \mathcal{T}$.

It is assumed that the integrals in (1) exist in the Pettis sense, which guarantees the $\sigma$-additivity of $b$ with respect to the first variable. The $\sigma$-additivity with respect to the second variable is not automatic, therefore it is assumed. It can be guaranteed (via the dominated convergence theorem) by the boundedness of the family of measures $\{m(\omega): \omega \in \Omega\}$ in a suitable sense.

EXAmple 1. Let $\Omega=\Upsilon=(0,1)$, let $\delta=\sigma$ be the Borel $\sigma$-algebra in $(0,1)$, $X=\mathbf{R}$,

$$
m(\omega ; F)=\int \frac{\omega^{2}-v^{2}}{F\left(\omega^{2}+v^{2}\right)^{2}} d v, \quad F \in \mathcal{T}
$$


for every $\omega \in \Omega$, and let $\lambda$ be the Lebesgue measure in $\Omega$. Then the integral in (1) exists for every $E \in \mathcal{S}$ and every $F \in \mathcal{T}$, but the resulting application $F$ $\mapsto b(\Omega, F), F \in \mathcal{T}$, is not $\sigma$-additive.

The representation (1) is already interesting in the case $X=\mathbf{R}$. We make $\mathrm{ca}(\mathcal{T}, \mathbf{R})$ a Banach space by endowing it with the usual total variation norm. If the integral

$$
\int_{\Omega} m(\omega) \lambda(d \omega)
$$

exists in the Bochner sense, then $b$, defined by (1), can be extended to a finite $\sigma$-additive measure on the whole of $\delta \otimes \mathcal{T}$. This is a situation corresponding to the classical Fubini-Tonelli theorem or, rather, to the Cavalieri principle. If (2) exists only in Pettis's sense, then, in general, $b$ does not generate a $\sigma$-additive measure on $\delta \otimes \mathcal{T}$.

Conversely, if (1) exists for every $E \in \mathcal{S}$ and $F \in \mathcal{T}$ and if the so defined function $b$ is a scalar bimeasure on $\mathcal{S} \times \mathcal{T}$, then the integral (2) exists in Pettis's sense. This follows from the mentioned Ylinen's theorem (Theorem 4.4 in [6]) saying that the evaluations on sets from $\mathcal{T}$ form a subset of $\operatorname{ca}(\mathcal{T}, \mathbf{R})^{\prime}$ with the Orlicz-Pettis property. This is a case occuring quite often.

EXAMPLe 2. Let $\Omega=\Upsilon=\mathbf{N}$ be the set of all natural numbers, $\delta=\mathcal{T}=\exp \mathbf{N}$, $X=\mathbf{R}$. For every $\omega \in \Omega$, let the sequence $v \mapsto f(\omega, v), v \in \Upsilon$, denoted by $f(\omega, \cdot)$, represent an element of $l^{1}$ and let

$$
m(\omega ; F)=\sum_{\nu \in F} f(\omega, v), \quad F \in \mathcal{T} .
$$

Assume that the sequence $\{f(\omega, \cdot): \omega \in \Omega\}$ of elements of $l^{1}$ is unconditionally but not absolutely summable. Let $\lambda$ be the 'counting' measure on $\mathfrak{S}$.

Then, clearly, the integral (2) exists as the Pettis integral but does not exist as the Bochner integral. Furthermore, (1) defines a bimeasure $b$ on $\mathcal{S} \times \mathcal{T}$ which does not generate a measure on $\mathcal{S} \otimes \mathcal{T}$.

Noting that the class of Pettis integrable functions is considerably larger than the class of Bochner integrable ones, we see that the described construction produces many bimeasures which are not measures. However, the construction is far from universal as can be seen in the following

EXAMPLE 3. Let $\Omega=\Upsilon=(0,1)$ and let $\delta=\sigma$ be the Borel $\sigma$-algebra. Let $X=L^{2}(0,1)$. Let $\alpha$ and $\beta$ be positive numbers such that $\alpha<1, \beta<1$ and $\alpha+\beta>3 / 2$. Given $E \in \mathcal{S}$ and $F \in \mathcal{T}$, let

$$
b(E, F)(t)=\int_{E}\left(\int_{E}|\omega-v|^{-\beta}|v-t|^{-\alpha} d v\right) d \omega, \quad t \in(0,1) .
$$

It is easy to see that $b(E, F)$, as a function on $(0,1)$, represents an element of $X$ and that the application $(E, F) \mapsto b(E, F), E \in \mathcal{S}, F \in \mathcal{T}$, is an $X$-valued bimeasure.

It is clear that, in the representation (1), if any, only a measure equivalent to Lebesgue measure can be taken for $\lambda$. The candidate for $m(\omega, T)$ is the function 


$$
t \mapsto \int_{\Upsilon}|\omega-v|^{-\beta}|v-t|^{-\alpha} d v, \quad t \in(0,1),
$$

or, if $\lambda$ is not the Lebesgue measure itself, a constant multiple of this function, $\omega \in \Omega$. However, it does not represent an element of $X$.

Nevertheless, the bimeasure $b$ is rather well-behaved. It can be extended to an

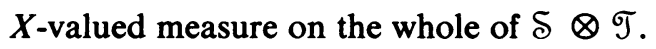

Many scalar valued bimeasures not admitting the representation (1) are obtained simply by composing the bimeasure $b$ with continuous linear functionals on $X$.

Let $X$ be a locally convex space. Let $Y$ be a locally convex space such that $X \subset Y$ and the inclusion map is a continuous injection. Let $b: \mathcal{S} \times \mathcal{T} \rightarrow X$ be a bimeasure. A family of measures $m(\omega) \in \mathrm{ca}(\mathcal{T}, Y), \omega \in \Omega$, is called a disintegration of the bimeasure $b$ in the space $Y$ with respect to a measure $\lambda \in \mathrm{ca}^{+}(\mathcal{\delta}, \overline{\mathbf{R}})$, if the equality (1) holds for every $E \in \mathcal{S}$ and $F \in \mathcal{T}$.

More properly, we should speak about a 'left' disintegration and introduce an analogous concept of 'right' disintegration. However, because of the symmetry, we discuss only one of these concepts and simplify the terminology accordingly.

For the bimeasure of Example 3 we can take $Y=L^{1}(0,1)$. The existence of a disintegration in $Y$ with respect to the Lebesgue measure is then clear.

Using a disintegration of the bimeasure $b$, we can express the integral of a function with respect to $b$ in a form similar to the classical Fubini theorem.

Proposition. Let $m(\omega), \omega \in \Omega$, be a disintegration of the bimeasure $b: \mathcal{S} \times \mathfrak{T} \rightarrow$ $X$ in the space $Y$ with respect to $\lambda \in \mathrm{ca}^{+}(\mathcal{S}, \overline{\mathbf{R}})$. Let $f$ be a scalar valued, $\mathcal{S} \otimes \mathcal{T}_{-}$ measurable function on $\Omega \times \Upsilon$ such that

(i) for every $\omega \in \Omega$, the function $v \mapsto f(\omega, v), v \in \Upsilon$, is $m(\omega)$-integrable;

(ii) for every $F \in \mathcal{T}$, the function

$$
\omega \mapsto \int_{E} f(\omega, v) m(\omega ; d v), \quad \omega \in \Omega
$$

is Pettis $\lambda$-integrable in the space $Y$; and

(iii) for every $E \in \mathcal{S}$ and $F \in \mathcal{T}$, the integral

$$
c(E, F)=\int_{E}\left(\int_{F} f(\omega, v) m(\omega ; d v)\right) \lambda(d \omega)
$$

belongs to $X$ and the resulting map $c: \mathcal{S} \times \mathcal{T} \rightarrow X$ is a bimeasure.

Then the function $f$ is $b$-integrable and $(f b)(E, F)=c(E, F)$, for every $E \in \mathcal{S}$ and $F \in \mathcal{T}$.

Proof. The proof follows by composing $b$ with continuous linear functions on $Y$. Because the inclusion of $X$ into $Y$ is injective, the dual space of $Y$ is total on $X$.

The assumptions and application of the Proposition are simplified if the dual of $Y$ represents a subset of the dual of $X$ with the Orlicz-Pettis property.

If $m: \mathcal{S} \rightarrow \operatorname{ca}(\mathcal{T}, \mathbf{R})$ is a measure valued measure and if we let

$$
\beta(E, F)=m(E)(F), \quad E \in \mathcal{S}, F \in \mathcal{T},
$$

then $\beta: \mathcal{S} \times \mathcal{T} \rightarrow \mathbf{R}$ is a scalar bimeasure. Conversely, Ylinen has proved [6] that every bimeasure $\beta$ : $\mathcal{S} \times \mathcal{T} \rightarrow \mathbf{R}$ is obtained in this way: if $\beta$ is a scalar bimeasure, then (3) defines a vector measure $m: \mathcal{S} \rightarrow \mathrm{ca}(\mathcal{T}, \mathbf{R})$. 
We say that a bimeasure $\beta: \delta \times \mathcal{T} \rightarrow \mathbf{R}$ has finite variation (on a set $E \in \mathcal{S}$ ) if the corresponding vector measure $m: \mathcal{S} \rightarrow \mathrm{ca}(\mathcal{T}, \mathbf{R})$ does. The variation of $\beta$ can, of course, be easily expressed directly in terms of $\beta$.

It is advantageous to go still further with the 'vectorization' of scalar bimeasures. We associate with a bimeasure $\beta: \mathcal{S} \times \mathcal{T} \rightarrow \mathbf{R}$ the $\mathrm{ca}(\mathcal{T}, \mathbf{R})$-valued bimeasure

$$
(E, F) \mapsto \chi_{F} m(E), \quad E \in \mathcal{S}, F \in \mathcal{T},
$$

where $m$ is the ca( $(T, \mathbf{R})$-valued measure defined by (3), and the element $\chi_{F} m(E)$ of ca $(\mathcal{T}, \mathbf{R})$ (the indefinite integral of $\chi_{F}$ with respect to $m(E)$ ) is obtained by annihilating the measure $m(E)$ off the set $F$ and leaving it intact inside $F$, for any $E \in \mathcal{S}$ and $F \in \mathcal{T}$.

We shall not distinguish between the scalar bimeasure $\beta$ and the vector bimeasure (4). In particular, we shall speak about disintegrations of $\beta$ instead of disintegrations of the bimeasure (4). This is, in fact, the main advantage of considering the bimeasure (4) instead of $\beta$. There is no possibility of extending the range space $\mathbf{R}$ of $\beta$ and weakening its topology so as to obtain a disintegration, while for the bimeasure (4) this can be done.

Given a bimeasure $\beta: \mathcal{S} \times \mathcal{T} \rightarrow \mathbf{R}$, the whole space ca $(\mathcal{T}, \mathbf{R})$ may not be needed to accommodate the values of the vector measure $m$ defined by (3) or the vector bimeasure (4). Without explicitly mentioning it, we may interpret the measure $m$ or the bimeasure (4) as taking values in a suitable subspace of $\mathrm{ca}(\mathcal{T}, \mathbf{R})$.

Given a compact Hausdorff space $\Upsilon$, the symbol $C(\Upsilon)$ denotes, as usual, the space of all continuous real valued functions on $\Upsilon$, and $M(\Upsilon)$ the dual of $C(\Upsilon)$. Besides the norm topology, the weak dual topology on $M(\Upsilon)$ will be of interest.

Let $\mathcal{T}$ be the Baire $\sigma$-algebra in a topological space $\Upsilon$. Given an increasing sequence of compact set $\Upsilon_{k} \in \mathcal{T}, k=1,2, \ldots$, let

$$
Y=\lim _{\leftarrow k} M\left(\Upsilon_{k}\right)
$$

denote the projective limit of the spaces $M\left(\Upsilon_{k}\right), k=1,2, \ldots$, each endowed with the weak dual topology. If $X$ be the subspace of ca $(\mathcal{T}, \mathbf{R})$ consisting of those measures which vanish on sets disjoint with each $\Upsilon_{k}, k=1,2, \ldots$, then there is a continuous injection of $X$ into $Y$. This is evident from the existence of natural projections of $X$ onto $M\left(\Upsilon_{k}\right), k=1,2, \ldots$, realized by restricting elements of $X$ to subsets of $\Upsilon_{k}$ and identifying the resulting Baire measures in $\Upsilon_{k}$ with elements of $M\left(\Upsilon_{k}\right)$.

THEOREM. Let $S$ be a $\sigma$-algebra of sets in a space $\Omega$ and $\mathcal{T}$ the $\sigma$-algebra of Baire sets in a topological space $\Upsilon$. Let $\beta: \mathcal{S} \times \mathcal{T} \rightarrow \mathbf{R}$ be a bimeasure.

Assume that $\Omega_{k}$ is an increasing sequence of sets from $\mathcal{S}$ and $\Upsilon_{k}$ an increasing sequence of compact sets from $\mathcal{T}$, the restriction of $\beta$ to any $\Omega_{k}$ and $\Upsilon_{k}, k=$ $1,2, \ldots$, has finite variation, and

$$
\lim _{k \rightarrow \infty} \beta\left(E \cap \Omega_{k}, F\right)=\beta(E, F)=\lim _{k \rightarrow \infty} \beta\left(E, F \cap \Upsilon_{k}\right),
$$

for every $E \in \mathcal{S}$ and $F \in \mathcal{T}$.

Let $Y$ be the projective limit of the spaces $M\left(\Upsilon_{k}\right), k=1,2, \ldots$

Then there exists a measure $\lambda \in \mathrm{ca}^{+}(\mathcal{S}, \mathbf{R})$ and a disintegration of $\beta$ with respect to $\lambda$ in $Y$. 
Proof. The proof follows in an almost routine way from the following

LEMMA. Let $\mathcal{S}$ be a $\sigma$-algebra of sets in a space $\Omega$, let $\Upsilon$ be a compact Hausdorff space, and $m: \mathcal{S} \rightarrow M(\Upsilon)$ a vector measure with finite variation $\lambda$. Then there exists a function $\Phi: \Omega \rightarrow M(\Upsilon)$ such that

$$
\int_{E} \Phi(\omega)(\varphi) \lambda(d \omega)=m(E)(\varphi)
$$

for every $E \in \mathcal{S}$ and $\varphi \in C(\Upsilon)$.

Proof. Clearly, $|m(E)(\varphi)| \leqslant \lambda(E)\|\varphi\|_{\infty}$, for every $E \in \mathcal{S}$ and $\varphi \in C(\Upsilon)$. Hence, if $\omega \mapsto \Psi(\omega ; \varphi), \omega \in \Omega$, is a version of the Radon-Nikodým derivative of the measure $E \mapsto m(E)(\varphi), E \in \mathcal{\delta}$, with respect to $\lambda$, then

$$
\|\Psi(\cdot ; \varphi)\|_{\lambda, \infty}=\lambda-\operatorname{ess} \sup \{|\Psi(\omega ; \varphi)|: \omega \in \Omega\} \leqslant\|\varphi\|_{\infty},
$$

for every $\varphi \in C(\Upsilon)$. By the Maharam theorem [3] on lifting in $L^{\infty}(\lambda)$, for every $\varphi \in C(\Upsilon)$, there exists an $\mathcal{S}$-measurable function $\omega \mapsto \Phi(\omega)(\varphi), \omega \in \Omega$, determining the same element of $L^{\infty}(\lambda)$ as $\Psi(\cdot ; \varphi)$, such that

$$
\sup \{|\Phi(\omega)(\varphi)|: \varphi \in \Omega\}=\|\Psi(\cdot ; \varphi)\|_{\lambda, \infty}
$$

and, for every $\omega \in \Omega$, the functional $\varphi \mapsto \Phi(\omega)(\varphi), \varphi \in C(\Upsilon)$, is linear. Because $|\Phi(\omega)(\varphi)| \leqslant\|\varphi\|_{\infty}, \varphi \in C(\Upsilon)$, this functional is continuous. Hence $\Phi(\omega) \in M(\Upsilon)$, for every $\omega \in \Omega$. The equality (5) is clear from the definition of $\Phi$.

There are obvious variants of the Theorem. For example, we can require the sets $\Omega_{k}$ and $\Upsilon_{k}, k=1,2, \ldots$, to be pair-wise disjoint instead of forming increasing sequences. With certain care, we can then allow uncountably many of the sets $\Omega_{k}$ at the cost of admitting infinite values for $\lambda$.

Instead of Baire sets in $\Upsilon$, we could take Borel sets adding some regularity requirements. However, it is not possible to dispense with all the topological assumptions or some variants of these. All the problems concerning disintegration of probability measures are inherent also in our present problems, and already the classical theorem of $\mathbf{M}$. Jiřina, on regular conditional probabilities, breaks down without some assumptions of a topological or similar nature. Questions of this kind are discussed, to the practical limits of possibilities, in [5].

Under the assumptions of the Theorem, the variation of the bimeasure $\beta$ may not be finite on any nonnegligible set $E \in \mathcal{S}$. Hence, the applicability of the Theorem is quite wide. It is reasonably easy to use. Let us consider the following classical

EXAMPLE 4. Let $l \geqslant 1$ be an integer. Let $\Omega=\Upsilon=\mathbf{R}^{l}$ and $\delta=\mathcal{T}$ be the Borel $\sigma$-algebra. Let $\varphi \in L^{2}\left(\mathbf{R}^{l}\right), \varphi \notin L^{2}\left(\mathbf{R}^{l}\right), \psi \in L^{2}\left(\mathbf{R}^{l}\right), \psi \notin L^{1}\left(\mathbf{R}^{l}\right)$, and

$$
p(\zeta)=(\pi i)^{-l / 2} \exp \left(i|\zeta|^{2}\right), \quad \zeta \in \mathbf{R}^{l} .
$$

For every set $E \in \mathcal{S}$, with finite Lebesgue measure, let

$$
m(E)(v)=\int_{E} \varphi(\omega) p(\omega-v) d \omega, \quad v \in \Upsilon
$$


It is easy to see (for example, using the Plancherel theorem) that $m(E) \in L^{2}\left(\mathbf{R}^{\prime}\right)$ and $\|m(E)\|=\left\|\chi_{E} \varphi\right\|\left(\right.$ norms in $L^{2}\left(\mathbf{R}^{\prime}\right)$ ). Hence, $m$ can be extended, in a unique way, to a measure $m: \delta \rightarrow L^{2}\left(\mathbf{R}^{l}\right)$.

For $E \in \mathcal{S}$ and $F \in \mathcal{T}$, let

$$
\beta(E, F)=\int_{F} m(E)(v) \overline{\psi(v)} d v .
$$

Then $\beta: \mathcal{S} \times \mathcal{T} \rightarrow \mathbf{R}$ is a bimeasure. It has infinite variation on every set $E \in \mathcal{S}$ of nonzero Lebesgue measure. The Theorem is, clearly, applicable by taking $\Omega_{k}$ and $\Upsilon_{k}$ to be, say, balls of radius $k=1,2, \ldots$.

\section{REFERENCES}

1. Claude Dellacherie and Paul-André Meyer, Probabilités et potentiel, Actualités Sci. Indust., no. 1372, Hermann, Paris, 1975.

2. Shizuo Kakutani, An example concerning uniform boundedness of spectral measures, Pacific J. Math. 4 (1954), 363-372.

3. Dorothy Maharam, On a theorem of von Neumann, Proc. Amer. Math. Soc. 9 (1958), 987-994.

4. E. Marczewski and C. Ryll-Nardzewski, Remarks on the compactness and non direct product of measures, Fund. Math. 40 (1953), 165-170.

5. Laurent Schwartz, Surmartingales régulières à valeurs mesures et désintégrations régulierès d'une mesure, J. Analyse Math. 26 (1973), 1-168.

6. Kari Ylinen, On vector bimeasures, Ann. Mat. Pura Appl. (4) 117 (1978), 115-138.

Department of Statistics, University of North Carolina at Chapel Hill, Chapel Hill, North Carolina 27514

Current address: The Flinders University of South Australia, Bedford Park, S. A., 5042, Australia 